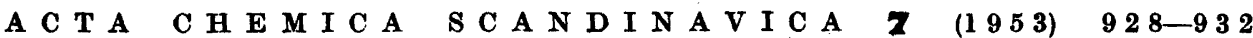

\title{
The Anomalous Electric Dipole Moment of p-Quinones
}

\author{
HELMER KOFO D* \\ Physical Chemistry Laboratory and Magdalen College, Oxford, England
}

\begin{abstract}
The electric dipole moment of 2,5-di-tert-butyl-1,4-benzoquinone has been measured in benzene solution at $25^{\circ} \mathrm{C}$. It is $0.81 \pm 0.03 \mathrm{D}$. The origin of the unexpectedly large apparent moments of $p$-benzoquinone and various symmetrical derivatives is discussed in the light of the new result.
\end{abstract}

The conventional formulation of $p$-benzoquinone is symmetrical. X-ray 1 work $^{1}$ has revealed that in the crystalline state the ring is an irregular hexagon, but the molecule including the oxygen atoms is coplanar and centrosymmetrical. It is reasonable to assume that the same structure prevails in dilute solution, because of inter alia the resonance in the system. Consequently $p$-benzoquinone should have a zero dipole moment. Hassel and Naeshagen ${ }^{2}$, however, found the value $0.67 \mathrm{D}$ in benzene solution. They suggested that the observed orientation polarisation is an apparent one, and that an abnormally high atom polarisation is responsible for it.

Hammick, Hampson and Jenkins ${ }^{3}$ put forward the hypothesis that the molecule including the ring is distorted out of plane by thermal impacts to polar forms, which should be retained long enough to give rise to an orientation polarisation. A calculation of the period of vibration, however, showed this to be much smaller than the time of relaxation, and the explanation was further weakened by observed dipole moments of substituted quinones. By extending the measurements to solvents other than benzene still the same polarisation was found, so that also an explanation on grounds of solvent effect was rendered unlikely. It was finally ruled out by Coop and Sutton ${ }^{4}$, who found practically the same value in the vapour phase and moreover observed temperature invariancy of the polarisation within a considerable range, which confirms that the thermal bending postulate was an invalid explanation. Atom polarisation, as suggested by Hassel and Naeshagen ${ }^{2}$, was therefore deemed to be responsible for the anomaly, as the only possibility left. The type of vibration giving rise to the abnormally high atom polarisation is probably not the one suggested by Hammick et $a l .^{3}$ as a part of the thermal bending hypo-

* Present address: Danmarks farmaceutiske Højskole, København. 
Fig. 1. Steric interference between the oxygen atom and ortho-substituents in benzoquinone. For the methyl and tert-butyl groups only one hydrogen contour is indicated in the position of maximum overlap.

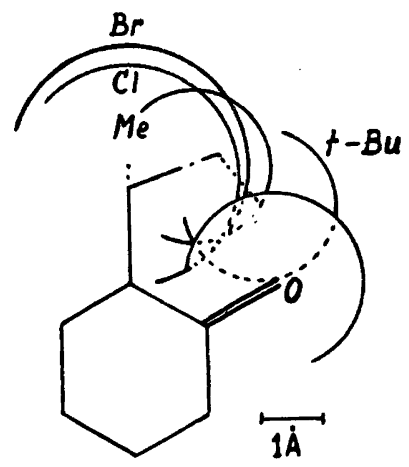

thesis, since the vibration period disagrees with that calculated from absorption frequencies in the infra red. The mode of vibration suggested by Coop and Sutton ${ }^{4}$ was the following. Each $\mathrm{C}=\mathrm{O}$ bond vibrates relative to the rest of the molecule in the plane of the ring i.e. perpendicularly to the plane of the $\pi$-bond orbitals. If this view is correct, a decrease of atom polarisation and hence of the apparent dipole moment should be expected, when the freedom of vibration is sterically decreased by the presence of bulky substituents in the ortho positions. Hammick et al.3, however, working along other lines, observed much the same moment, within the experimental accuracy, of a number of substituted quinones (vide Table 1).

In 1,4-di-tert-butyl-2,5-benzoquinone the steric interference between adjacent groups is much larger than in any other compound listed in Table 1 (cf. the scale diagram, Fig. 1). This substance was at hand as an intermediate in some synthetic research. It has been measured in the hope of throwing further light on the problem of the quinone moment.

The results are recorded in the bottom line of Table 1 together with the earlier measurements by the authors quoted.

Table 1. Polarisations of substituted benzoquinones in benzene solution at $25^{\circ} \mathrm{C}$.

\begin{tabular}{l|r|r|r|r|r}
\hline & $T P_{\text {vap. }}{ }^{*}$ & $T P$ & $R_{\mathrm{D}}$ & $T P-R_{\mathrm{D}}$ & $\mu_{\text {app. }}$ \\
\hline 1,4-Benzoquinone & 36.6 & 37.1 & 28.3 & 8.8 & 0.65 \\
2,5-Dichloro-1,4-benzoquinone & 46.3 & 47.2 & 38.4 & 8.8 & 0.65 \\
2,5-Dimethyl-1,4-benzoquinone & 47.4 & 47.8 & 38.4 & 9.4 & 0.68 \\
2,5-Dibromo-1,4-benzoquinone & & 54 & 44.0 & 10 & 0.7 \\
2,5-Di-tert-butyl-1,4-benzoquinone & & 79.7 & 66.3 & 13.4 & 0.81 \\
& & & & & 0.03
\end{tabular}

* Coop and Sutton "; bottom line by the present author; all other values by Hammick et $a .^{3}$

The substances in Table 1 are arranged in the order of increasing steric interference, applying the usual van der Waals radii and atomic distances ${ }^{5}$, cf. Fig. 1. ${ }_{T} P_{\text {vap. is the total polarisation in the vapour phase, }{ }_{T} P \text { is the corre- }}$ sponding term in benzene solution. The refractivities $R_{\mathrm{D}}$ are experimental

Acta Chem. Scand. 7 (1953) No. 6 
values except for the bromo compound, for which it was calculated from atom refractivities. ${ }_{T} P-R_{\mathrm{D}}$ thus represents the apparent orientation polarisation with the conventional minor correction for atom polarisation implied in not extrapolating to infinite wave length.

It is evident from the table that the introduction into the quinone of the two tert-butyl groups does not diminish its electric moment, on the contrary it is slightly increased. The increase is too small relative to the experimental accuracy to justify quantitative treatment, but the following conclusions may probably be drawn from the results.

If the apparent moment of $p$-benzoquinone is due to abnormally high atom polarisation, then this is unaffected by even very voluminous groups as tertbutyl. Since the envelope of the freely rotating groups must have a considerable overlap with the oxygen atoms, vide Fig. 1, the hypothesis must involve that the rotation of the butyl group is stopped, and it is fixed in a position of minimum interference. Such an arrangement can be realized without appreciable strain.

An alternative hypothesis is that atom polarisation involving the oxogroups is responsible only for the moment of benzoquinone itself, whereas this effect is sterically suppressed in at least some of the substituted compounds. The apparent moment of those should then be due to other reasons. The rise in ${ }_{T} P-R_{\mathrm{D}}$ of the butyl-compound over the value of the unsubstituted quinone is $4.6 \mathrm{~cm}^{3}$. It cannot be explained solely as an atom polarisation of the tertbutyl groups since ${ }_{T} P-R_{\mathrm{D}}$ for 1,4-di-tert-butyl-benzene was found to be as small as $1.6 \pm 0.5 \mathrm{~cm}^{3}\left(\operatorname{Kofod}\right.$ et $\left.a .^{6}\right)$. Subtracting the atom polarisation of the benzene ring, $0.4 \mathrm{~cm}^{3}$, from the upper limit we get the maximum value $1.7 \mathrm{~cm}^{3}$ as the contribution of the butyl groups in that compound. 1.7 is likely to be valid as a maximum value also in di-tert-butyl-benzoquinone on the assumption that the steric interference reduces the freedom of vibration of interfering groups. One therefore has to explain an apparent orientation polarisation of at least 2.9, which is well beyond the experimental error. Induced moments are no doubt operative in the molecule, but they will almost certainly cancel out because of the symmetry of the molecule. Neither does an explanation on grounds of solvent-solute interaction seem to be plausible, because it should be most marked in connection with the polar-oxo groups, much less with the butyl groups, and benzoquinone itself does not show any solvent effect. Finally the exaltation could be explained as a true orientation polarisation if the interfering groups or some of them were permanently deflected out of the ring plane, or if the entire ring was distorted, giving rise to polar forms. The latter distortion is unlikely because of the resonance in the molecule and since it is perfectly planar in the crystalline state. The former type of distortion, again because of the pronounced resonance in the quinone system, probably would mean deflection of chiefly the butyl groups out of plane. Their group moment is hardly big enough to account for the experimental facts, and secondly, if they were deflected out of plane it would most likely happen in a symmetrical way with no effect at all on the dipole moment. Although the compounds are not strictly analogous, it may be mentioned that in 2,5-di-bromo-1,4-di-tert-butylbenzene it was concluded 6 that either no deflection out of plane occurred or, if so, centrosymmetrical 
forms predominated, since the dipole moment of the compound was indistinguishable from zero.

The author is thus inclined to favour the former hypothesis, according to which abnormally high atom polarisation is responsible for the apparent dipole moment of both benzoquinone and the substituted compounds. The atom polarisation is due to vibrations of the oxo groups possibly including distortion of the ring and it is practically unaffected by the presence of the substituents $\mathrm{Cl}, \mathrm{Br}, \mathrm{CH}_{3}$ and $\left(\mathrm{CH}_{3}\right)_{3} \mathrm{C}$. The free rotation of the tert-butyl group is either stopped or we arrive at the same conclusion as in a previous paper ${ }^{6}$ that the conventional van der Waals radii are too large in this context. It is intended to measure duroquinone for comparison.

\section{EXPERIMENTAL}

Benzene. Analytical grade benzene was frozen out three times, about one-fifth being poured off and rejected each time, dried over phosphorus oxide, distilled just before use, and stored under dry air; it had f.p. $5.2^{\circ} \mathrm{C}$.

2,5-Di-tert-butyl-1,4-benzoquinone. The corresponding di-tert-butyl-hydroquinone was prepared by alkylation of hydroquinone with isobutyl alcohol using oleum as a dehydrating agent, according to Verley ${ }^{7}$ (cf. Smyth et al. $\left.{ }^{8}\right)$. The substituted hydroquinone was oxidized by suspending 50 grammes of the finely ground product in $300 \mathrm{ml}$ dilute nitric acid, 1 part concentrated and 3 parts water. The suspension was heated to the boiling point with frequent stirring for three hours after the colour of the solid had changed into bright yellow, and the evolution of nitrogen oxides ceased. The quinone was collected on a filter, washed with water and recrystallized from benzene. M.p. $150-151^{\circ}$, identical with that observed by E. Boedtker ${ }^{\circ}$, who obtained this compound in a different way. The identity was further established by reduction in alkaline medium with hydroxyl. amine, which led back to the di-tert-butylhydroquinone.

Physical measurements. All measurements were made at $25^{\circ} \mathrm{C}$. The electric dipole moment was obtained from the equation $\mu=0.2212\left(T P-R_{\mathrm{D}}\right)^{\frac{1}{2}}$. No correction was made for atom polarisation apart from that implied in using $R_{\mathrm{D}}$ to give the distortion polarisation. The total polarisation $T P$ was computed from observed values of dielectric constant and the specific volume $v$ for benzene solutions by Halverstadt and Kumler's procedure ${ }^{10}$. Symbols have the same significance as in their paper. The dielectric constants $\varepsilon$ were measured with a heterodyne beat capacity meter designed by Hill and Sutton. Specific volumes were measured with a modified Sprengel-Ostwald pyknometer. The molar refractions $R_{\mathrm{D}}$ were computed from the refractive indices, $n_{\mathrm{D}}$ and specific volumes. The refractive index $n_{\mathrm{D}}$ of a solution relative to that of the benzene used for preparing it was measured with a Pulfrich refractometer fitted with a centrally divided cell. Even the most concentrated solution had $n_{\mathrm{D}}$ identical with that of the pure solvent. The standard dielectric constant of pure dry benzene was taken as $2.2727^{11}$. The results are given in Table 2.

$\begin{array}{cccc}\text { Table 2. } & 2,5 \text {-di-tert-butyl-1,4-benzoquinone. } \\ 16^{5} \omega & \varepsilon & V & 10^{5} \Delta n_{\mathrm{D}} \\ 1020 & 2.2755 & 1.14365 & 0 \\ 920 & 2.2755 & 1.14379 & 0 \\ 870 & & 1.14384 & 0 \\ 573 & 2.2746 & 1.14418 & \\ 221 & 2.2731 & 1.14470 & \\ \varepsilon=2.2725+0.314 \omega ; & v=1.14494-0.127 \omega ; T P=79.7 ; R_{\mathrm{D}}=66.3 ; \mu=0.81 \pm 0.03 \quad \mathrm{D} .\end{array}$

The investigation was carried out during a two year stay at Oxford University. The author is greatly indebted to Dr. L. E. Sutton, F. R. S. for helpful advice and discussion and to The British Council for a scholarship.

Acta Chem. Scand. 7 (1953) No. 6 


\section{REFERENCES}

1. Robertson, J. M. Proc. Roy. Soc. A 150 (1935) 106.

2. Hassel, O., and Naeshagen, E. Z. physik. Chem. B 6 (1929) 441.

3. Hammick, D. Ll., Jenkins, G. I., and Hampson, G. C. J. Chem. Soc. 19381263.

4. Sutton, L. E., and Coop, I. E. J. Chem. Soc. 19381269.

5. Pauling, L. Nature of the Chemical Bond, New York 1945.

6. Kofod, H., Kumar, L., and Sutton, L. E. J. Chem. Soc. 19511794.

7. Verley, A. Bull. soc. chim. France [3] 19 (1898) 72.

8. Smyth, C. P., Oesper, P. F., and Kharasch, M. S. J. Am. Chem. Soc. 64 (1942) 937.

9. Boedtker, E. Bull. soc. chim. France [3] 31 (1904) 971.

10. Halverstadt, I. F., and Kumler, W. D. J. Am. Chem. Soc. 64 (1942) 2988.

11. Hartshorn, L., and Oliver, D. A. Proc. Roy. Soc. (London) $123 A$ (1929) 664.

Received May 8, 1953. 